\title{
Gaenslen's Split Heel Incision for Calcaneal Osteomyelitis: A case report
}

\author{
by J. Terrence Jose Jerome, MBBS, DNB (Ortho), MNAMS (Ortho) ${ }^{1} \mathbb{a}$, Mathew Varghese, M.S. (Ortho) ${ }^{2}$ \\ , Balu Sankaran, FRCS, FAMS ${ }^{3}$, Simon Thomas, MBBS, DNB (Ortho), MNAMS (Ortho) ${ }^{4}$
}

The Foot and Ankle Online Journal 2 (4): 3

Patients who have a recurrent ulcer of the heel often have a below-the-knee amputation because durable soft tissue coverage cannot be obtained over the calcaneus. Often, even if the ulcer heals satisfactorily or the area appears to be revascularized as a result of a peripheral vascular procedure, the resulting surface is not durable and the ulcer recurs. We report a 40 year old lady who had a chronic osteomyelitis right calcaneum with a large non-healing ulcer and discharging sinus, treated by split heel incision of Gaenslen.

Key words: Calcaneal osteomyelitis, Gaenslen’s incision

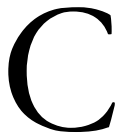

hronic osteomyelitis of the calcaneum presents a formidable management dilemma. Split-thickness skin grafts that are placed directly over the calcaneus occasionally can be used to cover the defect, but these grafts usually cannot withstand the forces that are generated during weight bearing. Furthermore, these patients are often poor candidates for a free tissue transfer because of underlying vascular disease or diabetes. We report a case in which Gaenslen's split-heel technique was successfully used in the management of calcaneal osteomyelitis. Gaenslen's technique is recommended for refractory cases or patients with a draining sinus centrally located on the plantar aspect of the heel. An excellent functional result can be obtained.

\footnotetext{
Address correspondence to: Dr. J. Terrence Jose Jerome, MBBS.,DNB (Ortho), MNAMS (Ortho)

Registrar in Orthopedics, Dept. of Orthopedics

St. Stephen's Hospital, Tiz Hazari, Delhi 54, India

${ }^{1}$ Registrar in Orthopedics, Department of Orthopedics, St. Stephens Hospital, Tiz Hazari, Delhi, India.

${ }^{2}$ Head Professor, Department of Orthopedics, St. Stephens Hospital, Tiz Hazari, Delhi, India.

${ }^{3}$ Professor Emeritus, Orthopedics, St. Stephens Hospital, Tiz Hazari, Delhi, India. E-mail: pasle@bol.net.in

${ }^{4}$ Registrar in Orthopedics, Department of Orthopedics, St. Stephens Hospital, Tiz Hazari, Delhi, India. Phone: 991-23966021-27.
}

A 40 year old lady with spina bifida came to our outpatient department with a large non-healing ulcer of her right heel for 3 months duration. She had consulted various doctors for the same problem, but could not get better. Her right heel showed a circular, $4 \mathrm{~cm}$ diameter, non-healing ulcer with seropurulent, draining sinus. (Figs. 1) The base of the ulcer had calcaneus. The margins and edges were ill-defined. The radiograph showed a lytic lesion of the posteroinferior calcaneus with a little break in the cortex. (Fig. 2) Erythrocyte sedimentation rate was $78 \mathrm{~mm} /$ hour (normal <14). Her blood parameters revealed hemoglobin $11.2 \mathrm{gms}$, TLC 7,500cu $\backslash \mathrm{mm}$. All other blood parameters were within normal limits. necrotic slough and was indurated to the underlying 

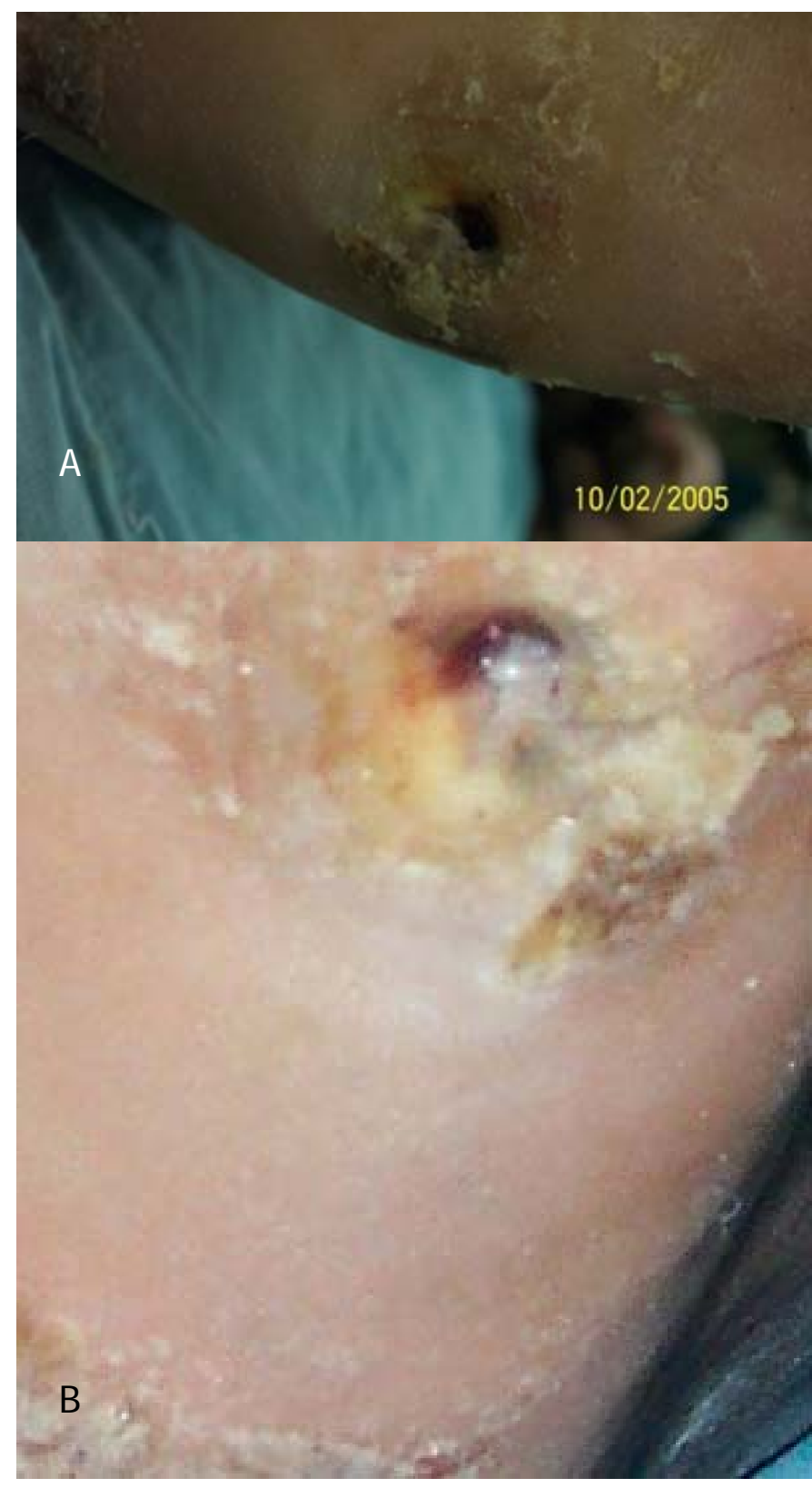

Figures 1 The patient's right heel showed a circular, 4 $\mathrm{cm}$ diameter, non-healing ulcer with seropurulent, draining sinus ( $A$ and $B$ ).

The patient was put in the prone position with a support beneath the right ankle. A longitudinal incision exactly in the midline of the heel, extending 2.5 to $4 \mathrm{~cm}$ from the level of the base of the fifth metatarsal posteriorly was performed to split the end of the tendocalcaneus.

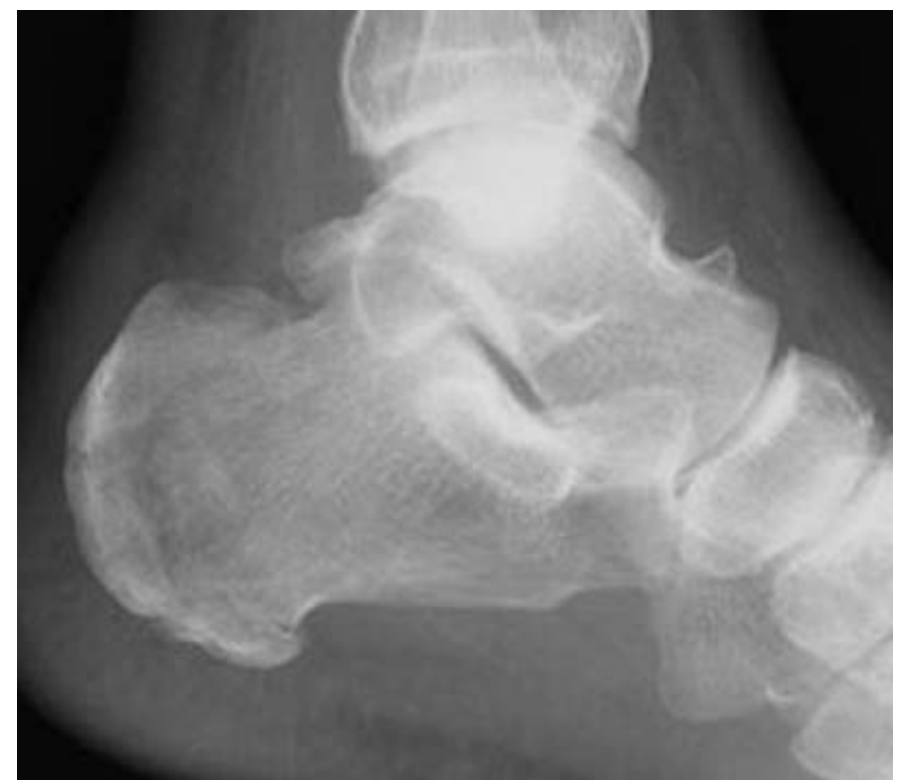

Figure 2 The radiograph showed a lytic lesion in the postero-inferior part with a little break in the cortex.

The plantar fascia was incised in the plane created between the abductor digiti quinti and flexor digitorum brevis. The lateral plantar artery was retracted medially and the nerve kept in the distal aspect of the wound. The quadratus plantae muscle and plantar ligament were split longitudinally. The calcaneus was visualized. It was divided from posterior to anterior with a broad osteotome and split into two halves to expose the interior of the bone (Fig 3). Sequestra, infected material, necrotic slough, damaged cortex were removed. Thorough lavage was performed.

The wound was closed loosely with a drain in-situ. A short leg cast was applied post-operatively with the foot in neutral position and ankle at 90 degree. A window was cut over the calcaneus to permit dressing changes. Sequential dressings were done every 4 th day. Two weeks after surgery, a pressure ulcer was noted just below medial malleolus (Fig 4). Extra padding and aseptic dressing was done. After 6 weeks, the pressure sore and the surgical wound healing was remarkable (Fig 5). The heel thus developed a deeply situated surgical scar over which the surrounding tissues were laid, forming thick cushions on either side of the incision. (Fig. 6) 


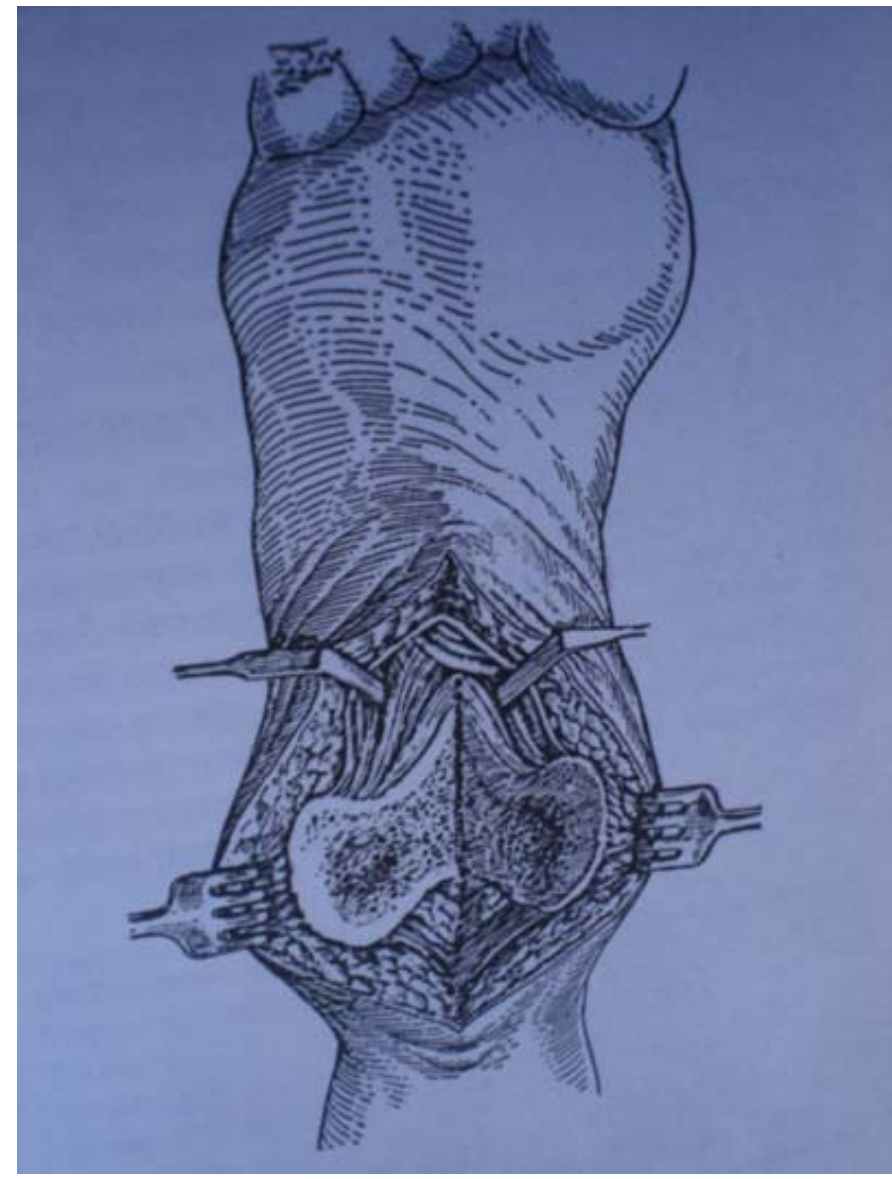

Figure 3 The Gaenslen approach uses a broad osteotome and splits the calcaneus into two halves to expose the interior of the bone.

\section{Discussion}

In osteomyelitis of the calcaneus, as of the other tarsal bones, the destruction of the cortex usually is not very extensive. The periosteum is firmly attached to the bone, and is usually perforated rather than elevated by purulent material; therefore the formation of involucrum is minimal. ${ }^{1}$ Partial calcanectomy is not a new procedure. Four reports, between 1931 and 1959, discussed resection of all or part of the calcaneus for the treatment of conditions as diverse as osteomyelitis of the calcaneus, cutaneous ulceration of the heel, and fracture of the calcaneus. ${ }^{2,3,4}$ These authors reported that all of the patients were managed successfully and maintained their functional mobility. Reports published between 1972 and 1991 also documented favorable results. ${ }^{1,5,6,7}$

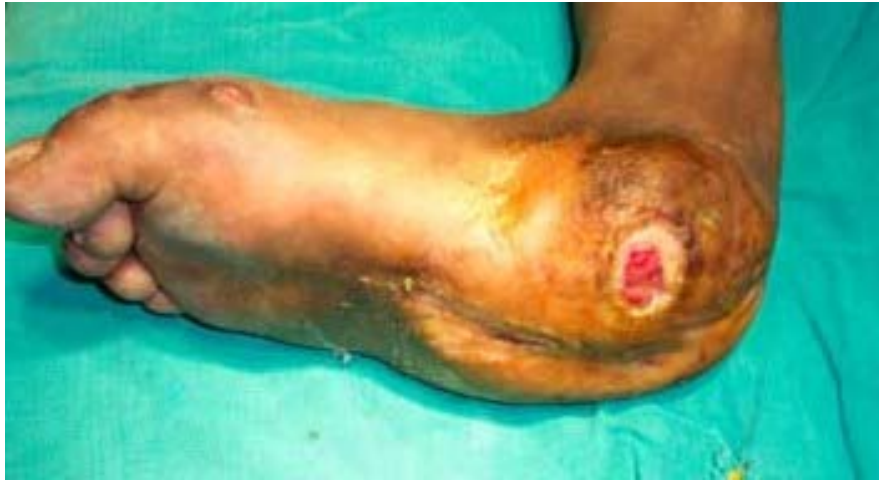

Figure 42 weeks after surgery, a small pressure ulcer developed just below the medial malleolus.

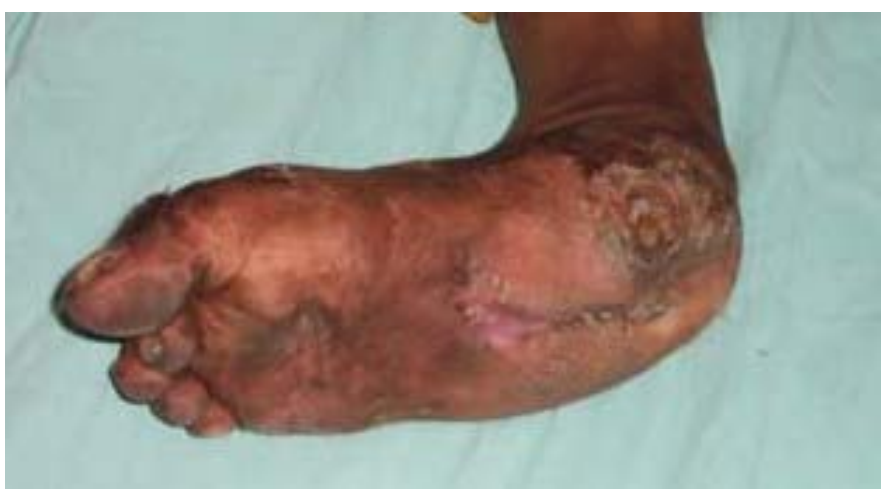

Figure 5 The surgical and pressure wounds healed without incident.

However, partial calcanectomy has not received much attention, and it seems that surgeons are often unaware that it is an option for the management of large ulcerations of the heel or osteomyelitis of the calcaneus.

Total calcanectomy is an alternative procedure to trans-tibial amputation in patients with chronic osteomyelitis of the calcaneus. Eradication of infection and preservation of functional ambulation is achieved. ${ }^{8}$ Assessment of ankle strength and range of motion of the surgical limb demonstrated decreased dorsiflexion and plantarflexion strength and variable range of motion compared to the contralateral limb. ${ }^{8}$ The split-heel technique was successfully used in the management of calcaneal osteomyelitis in children. 


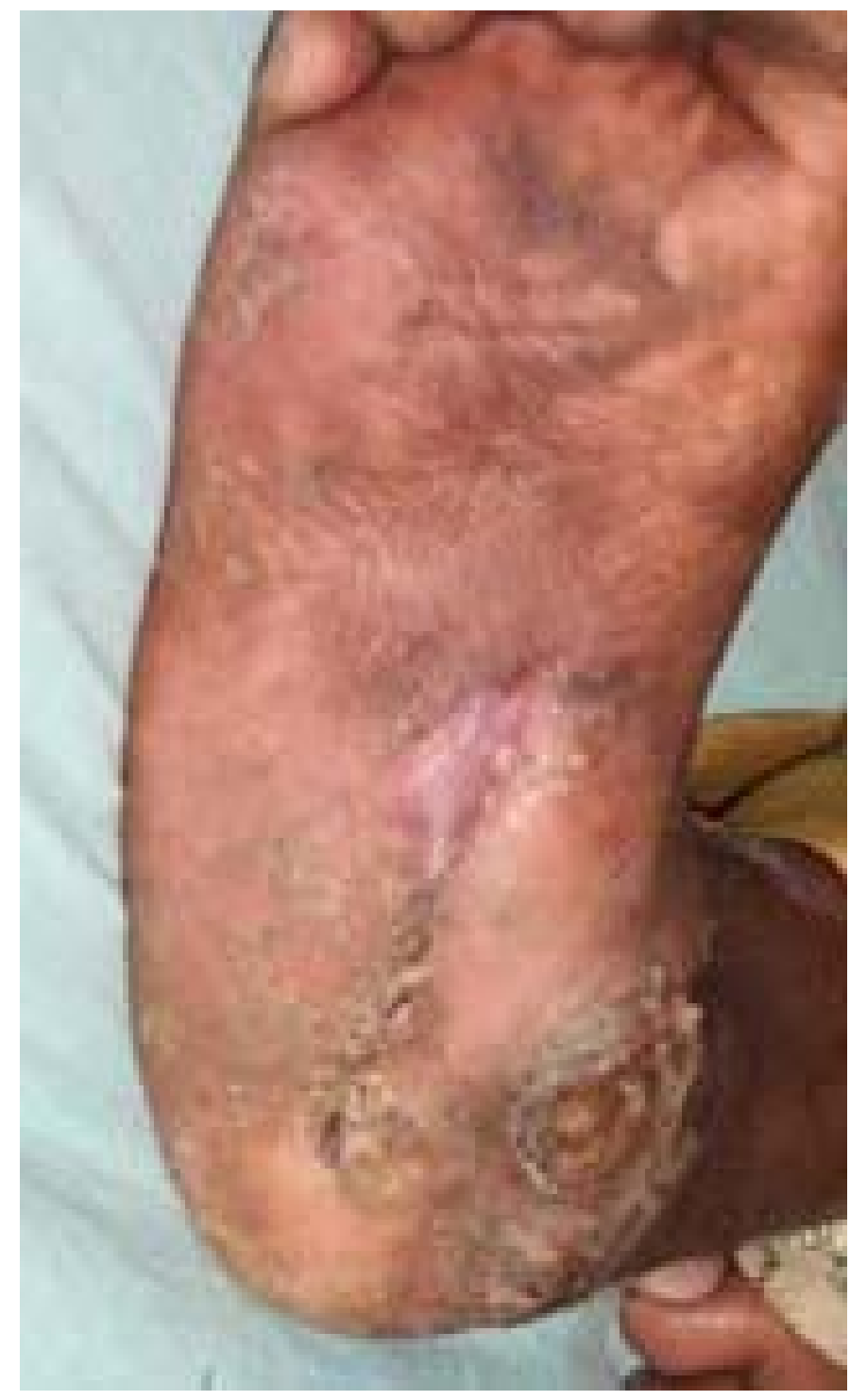

Figure 6 The heel develops a deeply situated surgical scar over which the surrounding tissues were laid, forming thick cushions on either side of the incision.

Antibiotic therapy and debridement when necessary through medial or lateral incisions will usually eradicate the infection.

Gaenslen's technique is recommended for refractory cases or patients with a draining sinus centrally located on the plantar aspect of the heel. In general, excision of the calcaneus for chronic osteomyelitis in children is not necessary. ${ }^{9}$ The resultant scars usually are painless.

(C) The Foot and Ankle Online Journal, 2009
They are so deeply situated that the tissues on either side curl inward. Diabetic, neuropathic patients are often at risk for ulceration. Those that are temporarily or permanently limited in ambulation and restricted to a supine position are, over time, very susceptible to heel decubiti. Subtotal calcanectomy is a relatively simple procedure to perform. Aggressive debridement of all nonviable tissue, thorough antibiotic course, ankle-foot orthosis combined with a custom-molded shoe in the postoperative period made all patients who were ambulatory preoperatively to resume their function after surgery. ${ }^{10}$ In the presence of adequate vasculature, it is a good alternative to below-the-knee amputation and the accompanying sequelae. Composite wounds of the heel with secondary osteomyelitis of the calcaneus remain a reconstructive challenge. A paucity of regional flaps has often necessitated microvascular transplantation in order to obtain wound healing. Subtotal calcanectomy, traditionally a technique of the orthopedic surgeon, can, in selected cases, eradicate infection and achieve wound closure and limb preservation. ${ }^{11}$

A distally based sural fascio-musculocutaneous flap including the sural nerve and a midline cuff of the gastrocnemius muscle can be useful for covering the defect. This flap is designed on the proximal half of the posterior calf and has an adequate blood supply derived from retrograde perfusion of the vascular axis of the sural nerve to the musculocutaneous perforators of the gastrocnemius muscle. The patency of the peroneal artery should be confirmed by doppler ultrasound or angiography before surgery. ${ }^{12}$ The flap was reliable and technically simple to design and execute. This 1-stage procedure not only preserves the major arteries of the injured leg but has also proved valuable for filling bony defect and treating bony infection because it provides a wellvascularized muscle fragment.

\section{Conclusion}

Our patient had a large non-healing plantar ulcer with discharging sinus along with chronic calcaneal osteomyelitis. Split heel incision of Gaenslen removed all sequestra, obviously infected matter and did little damage to the cortex. 
The resultant scar was painless and was so deeply situated that the tissues on either side curl inward to form 'cushions'. Gaenslen's technique is recommended for refractory cases of osteomyelitis or patients with a draining sinus centrally located on the plantar aspect of the heel. An excellent functional result can be obtained.

\section{References}

1. Antoniou D, Conner AN: Osteomyelitis of the calcaneus and talus. J Bone Joint Surg 56A: 338, 1974.

2. Gaenslen, FJ: Split-heel approach in osteomyelitis of os calcis. J. Bone joint Surg 13A: 759 - 772, 1931.

3. Wiltse LL, Bateman J, G, Kase S: Resection of major portion of the calcaneus. Clin Orthop 13: 271 - 278, 1959.

4. WoII TS, Beals RK: Partial calcanectomy for the treatment of osteomyelitis of the calcaneus. Foot and Ankle 12: 31 - 34, 1991. 5. Crandall RC, Wagner FW Jr: Partial and total calcanectomy. A review of thirty-one consecutive cases over a ten-year period. J Bone Joint Surg 63A: 152 - 155, 1981.

6. Horwitz T: Partial resection of the os calcis and primary closure in the treatment of resistant large ulcers of the heel with or without osteomyelitis of the os calcis. Clin Orthop 84: 149 $153,1972$.

7. Martini M, Martini-Benkeddache Y, Bekhechi T, Daoud A: Treatment of chronic osteomyelitis of the calcaneus by resection of the calcaneus. A report of twenty cases. J Bone Joint Surg.. 56A: $542-548,1974$.

8. Baumhauer JF, Fraga CJ, Gould JS, Johnson JE: Total calcanectomy for the treatment of chronic osteomyelitis, Foot Ankle 19: 849 - 851, 1948.

9. Broudy AS, Scott RD, Watts HG: The split heel technique in the management of calcaneal osteomyelitis in children. Report of three cases. Clin Orthop Relat Res (119): 202 - 5, 1976.

10. Baravarian B, Menendez MM, Weinheimer DJ, Lowery C, Kosanovich R, Vidt L: subtotal calcanectomy for the treatment of large heel ulceration and calcaneal osteomyelitis in the diabetic patient. J Foot Ankle Surg 38(5): 373 - 4, 1999.

11. Isenberg JS, Costigan WM, Thordarson DB: Subtotal calcanectomy for osteomyelitis of the os calcis: a reasonable alternative to free tissue transfer. Ann Plast Surg 35 (6): 660 - 3, 1995.

12. Chen SL, Chen TM, Chou TD, Chang SC, Wang HJ: Distally based sural fascio musculo cutaneous flap for chronic osteomyelitis in diabetic patients. Ann Plast Surg 54 (1):44 - 8, 2005 . 